\title{
DESIGN OF A CAPILLARY GRIPPER FOR A SUBMILLIMETRIC APPLICATION
}

\author{
Pierre Lambert \\ Université libre de Bruxelles CP 165-14 \\ Avenue F.D. Roosevelt, 50 \\ B - 1050 Bruxelles \\ pierre.lambert@ulb.ac.be
}

Frank Seigneur, Sandra Koelemeijer and Jacques Jacot

Ecole Polytechnique Fédérale de Lausanne

STI IPR LPMI - Station 17

$\mathrm{CH}$ - 1015 Lausanne

frank.seigneur@epfl, sandra.koelemeijer@epfl.ch, jacques.jacot@epfl.ch

\begin{abstract}
This paper describes the study of a gripper using the surface tension effects to pick and place the $0.5 \mathrm{~mm}$ or $0.3 \mathrm{~mm}$ diameter balls of a millimetric watch bearing. Two liquid supply strategies have been tested (pressure drive and tip dip). The effects of the coating (through the measurement of the contact angles), the presence of an internal channel and the size of the gripper have been studied. Analytical and numerical force models have been developped and validated thanks to a test bed allowing the measurement of the developped force (typically of the order of $100 \mu \mathrm{N}$ ) with a resolution of $1 \mu \mathrm{N}$. A complete pick and place cycle has been performed using the $0.5 \mathrm{~mm}$ diameter gripper. Such a test has still to be done in the future with the $0.3 \mathrm{~mm}$ diameter gripper.
\end{abstract}

Keywords: Microassembly, capillary forces, surface tension, submillimetric application

\section{INTRODUCTION}

This study aims at using the surface tension effects in the handling of submillimetric parts (the balls of a millimetric bearing). First, a brief state of the art is presented in section 2, highlighting the previous work concerning the surface tension based gripping. The proposed case study is detailed in section 3 , showing the design aspects. Then section 4 presents two new models to compute the force (they are adapted from existing ones to this case study). Section 5 summarizes the inputs of the models. Then, in section 6 , a functional val- 
idation of the gripper is presented and some force measurements confirm the proposed models. Finally, conclusions are drawn in section 7.

\section{STATE OF THE ART}

Within the framework of microassembly (and in particular in microgripping), the classical way consists in downscaling the two-fingered tweezer principle. This leads to considerable difficulties therefore a lot of new gripping principles has been proposed these last years: Lambert, 2004 refers to some existing reviews. These new principles take advantage of the scaling laws, because the forces involved decrease slower than the weight of the submillimetric components. They consequently represent an innovative alternative to miniaturized tweezers.

One of these principles, namely the capillary gripping, seems very promising as an alternative to miniaturized tweezers or vacuum pipets: indeed, this kind of gripping is well adpated to flat components with only free accessible surface (typically SMD components) but can also pick up more complex geometries (rings, balls). Its scaling law is very promising since the capillary force is directly proportional to the linear dimension $(F \div l)$, i.e. this principle generates forces larger than the weight $\left(W \div l^{3}\right)$ and is more efficient than the vacuum gripping $\left(F \div l^{2}\right)$ for the handling of submillimetric components. Moreover, as indicated in Grutzeck and Kiesewetter, 1998, there exists a damping effect which prevents mechanical damage due to high contact pressures.

This principle has already been proposed by Grutzeck and Kiesewetter, 1998 and Bark, 1999: they essentially validated the concept by handling millimetric silicon components. Lambert, 2004 deeply studied this gripping principle by modelling, numerical simulation and experimental validation. More recently Biganzoli et al., 2005 and Obata et al., 2004 proposed two different solutions in relation with an applicative framework: Biganzoli suggested to modify the gripper curvature while Obata tuned the volume of liquid.

\section{CASE STUDY}

The chosen case study deals with the design of a gripper to be used in the insertion of small balls (diameter $500 \mu \mathrm{m}$ ) in a hole. One of the requirements is to avoid the conventional tweezers and vacuum grippers, because of the scratches they provoke on the balls. Due to the very small weight of the balls (about $3.8 \mu \mathrm{N}$ ), the surface tension based gripper is largely strong enough since it generates forces up to $150 \mu \mathrm{N}$. The handling scheme is illustrated in figure 1: the picking force is provided by the capillary force and the releasing task is ensured by laterally moving the gripper once the ball is in the hole.

Since the gripper uses capillary forces, a liquid has to be dispensed before each manipulation, but there is no need to eject the liquid (such as for example 


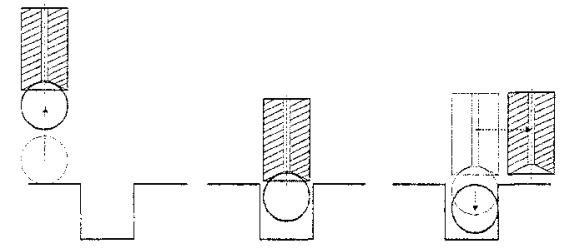

Figure 1. Handling scheme of the capillary gripping for the insertion of a ball in a hole.

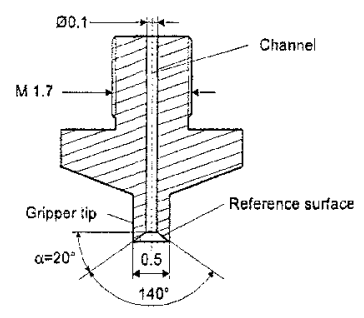

Figure 2a. Schematic view of the designed gripper: the so-called reference surface is the surface which contacts the ball during the handling.

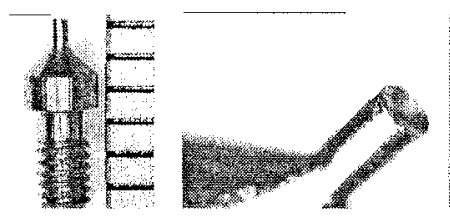

Figure 2b. Prototype (the distance between the graduations is $1 \mathrm{~mm}$ ) and detail of the conical tip.

in ink jet printing): it is sufficient to bring a bit of liquid in contact with the ball to pick up. Beside this dispensing functionnality, the other functions of the developped gripper tip can be summarized as follows: (1) to develop a picking force larger than the weight of the object $(W \approx 3.8 \mu \mathrm{N})$; (2) to develop a picking force large enough to handle the component with reasonable accelerations (manual handling); (3) to center the ball with respect to the gripper in order to ensure its positioning; (4) to release the ball once it is inserted in the hole. The proposed gripper is shown in figure $2 \mathrm{~b}$. It has been machined in stainless steel. Other prototypes have been coated with a hydrophobic silane-based coating.

Two solutions have been tried to supply the gripping liquid: (1) to drive the pressure through the gripper channel; (2) to dip the tip in the liquid.

Finally, the first solution has been discarded because of the instabilities of the droplet when its height approaches a half diameter of the gripper. It has been taken advantage of the good repeatability of the volume transferred to the gripper by dipping it to the liquid. For a large variety of grippers (see table 1), the ratio of the droplet height $h$ to the gripper diameter $D$ has been found to be 0.304 , with a standard deviation of 0.020 and a maximal residual of 0.061 .

\section{MODELS}

The force model discussed in this section can be viewed as a black box, as shown in figure 3 . The theoretical capillary force developped by the gripper has 


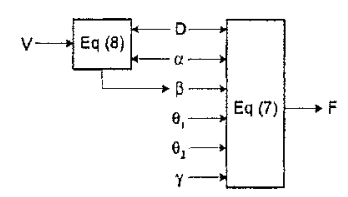

Figure 3. Schematic views of the models: $V$ is the dispensed volume of liquid, $D$ is the ball diameter ( $R$ is the radius), $\alpha$ is a geometrical parameter of the gripper, $\beta$ is the filling angle, $\theta_{1}$ and $\theta_{2}$ are the contact angles and $\gamma$ is the surface tension.

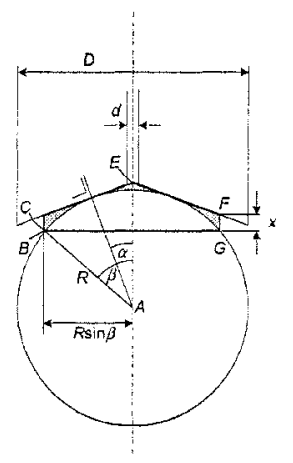

Figure 4a. Geometrical details of the gripper tip.

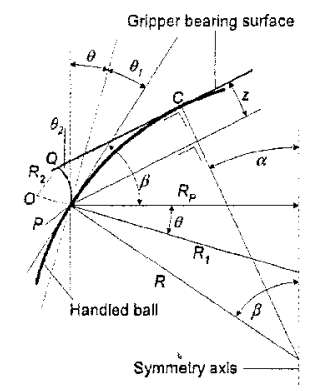

Figure $4 b . \quad$ Geometrical details of the meniscus.

been computed with an analytical model based on the so-called circle approximation of the meniscus geometry (the detailed problem is shown in figure $4 \mathrm{~b}$ ) and with a numerical model based on the Laplace equation, derived from that one presented in Lambert and Delchambre, 2005. The latter model is based on equations that were first proposed by Orr et al., 1975.

The situation depicted in figure $4 \mathrm{~b}$ shows that the meniscus wets the ball along the circle containing $P$ (the position of this circle, whose radius is equal to $R_{P}$, is determined by the filling angle $\beta$ ).

The contact angle $\theta_{1}$ is the angle between the tangent to the meniscus on the one hand and the tangent to the ball on the other hand: it is determined by the wetting properties of the materials, i.e. it is determined by the triple \{handling liquid, material of the ball, surrounding environment $\}$. On the gripper side (point $Q$ ), since the gripper and the ball are made of different materials, the contact angle $\theta_{2}$ can be different from $\theta_{1}$. The so-called circle approximation assumes a circular shaped meniscus: in the case of figure $4 \mathrm{~b}$, this circle is centred in $O$ and has a radius $R_{2}$. The capillary force can be written as:

$$
F=\pi R \sin ^{2} \beta \gamma\left[\frac{\sin \left(\beta+\theta_{1}\right)}{\sin \beta}+\frac{\cos \theta_{2}+\cos \left(\theta_{1}+\beta-\alpha\right)}{1-\cos (\beta-\alpha)}\right]
$$




\begin{tabular}{c|c|c|c|c|c|c|c|c}
\hline Gripper & $\begin{array}{c}D \\
(\mathrm{~mm})\end{array}$ & Channel & Coated & $\begin{array}{c}\theta_{A} \\
\left({ }^{\circ}\right)\end{array}$ & $\begin{array}{c}\sigma_{\theta_{A}} \\
\left({ }^{\circ}\right)\end{array}$ & $\begin{array}{c}\theta_{B} \\
\left({ }^{\circ}\right)\end{array}$ & $\begin{array}{c}\sigma_{\theta_{R}} \\
\left({ }^{\circ}\right)\end{array}$ & $\frac{h}{D}$ \\
\hline $\mathrm{B}$ & 0.5 & Yes & Yes & 22.7 & 4.2 & 19.3 & 3.5 & 0.287 \\
\hline $\mathrm{F}$ & 0.5 & Yes & No & 21.7 & 4.2 & 18.8 & 2.0 & 0.293 \\
\hline $\mathrm{H}$ & 0.5 & No & No & 21.0 & 2.6 & 16.8 & 2.5 & 0.305 \\
\hline $\mathrm{J}$ & 0.3 & No & Yes & 29.8 & 3.5 & 24.8 & 2.9 & 0.329 \\
\hline
\end{tabular}

Table 1. Properties of the tested grippers: $D$ is the gripper diameter, 'channel' denotes the presence of an internal chamel, 'coated' denotes the presence of a silane-based coating, $\theta_{A}$ is the advancing contact angle, $\theta_{R}$ is the receding contact angle and $\frac{h}{d}$ is the ratio between the height $h$ of the hanging droplet after the gripper has been dipped in liquid and $D$.

$$
+2 \pi R \sin \beta \gamma \sin \left(\beta+\theta_{1}\right)
$$

(this equation gives the force as a function of the handling liquid $(\gamma)$, the materials $\left(\theta_{1}, \theta_{2}\right)$, the size of the ball (radius $R$ ), the volume of liquid through the filling angle $\beta$ and the gripper geometry $(\alpha)$ ). One may notice that the meniscus is not defined for $\beta=\alpha$, leading to non physical result for the force (the force tends towards infinity).

The link between the dispensed volume of liquid $V$ and the filling angle $\beta$ can be determined as follows: in figure $4 \mathrm{a}$, the dispensed volume of liquid fills the $B C E F G$ area between the ball and the gripper, therefore:

$$
V=V_{1}-V_{2}
$$

where $V_{1}$ is the sum of the volumes of the cylindre $B C F G$ and the cone $C E F$, and $V_{2}$ is the volume of is the volume of the spherical cap of the ball limited by the filling angle $\beta$.

\section{EXPERIMENTAL SET UP}

The experimental set up used in this study has already been described in Lambert, 2004. It allows to measure the advancing and receding contact angles and to measure the capillary force with a resolution of the order of $1 \mu \mathrm{N}$. Four grippers have been tested whose properties are summarized in table 1 (the liquid which was used was a lubrication oil with a surface tension $\gamma=$ $34.5 \mathrm{mNm}^{-1}$. Note that oil has been used because it does not evaporate during the experiments. Moreover, since it can be the lubrication oil of the assembled device, it is not a drawback to have residual traces of liquid on the component after the manipulation). The balls are made in $\mathrm{Z}_{r} \mathrm{O}_{2}$.

\section{RESULTS}

Figure 5 illustrates an assembly cycle which has been achieved with the test bed mentionned in the previous section. On the first image, the ball to 
and Jacques Jacot

handle can be seen on the right side, 'floating' on a droplet of liquid (it 'floats' thanks to the surface tension effect, like the water strider, and not due to the Archimedes principle). On the left side of the image there is the bearing in which the ball has to be placed. On the second image, the gripper is aligned above the ball. The third image illustrates the picking of the ball, which is then moved (images 4,5 and 6) to its final location inside the bearing. The seventh image illustrates the radial shift of the gripper required to perform the release task. Finally, the gripper is moved away from the bearing.

Concerning the force models, the results of both models (analytical and numerical) are plotted in figures $6 a$ and $6 b$. On each figure, both the force and the volume of liquid between the gripper and the component are plotted as a function of the filling angle $\beta$. Two regimes can be distinguished: for $\beta<\alpha$ (here $\alpha=20^{\circ}$ ), the force is increasing with $\beta$ while the volume of liquid remains quite constant. In the case $\beta>\alpha$, the volume of liquid quickly increases with the filling angle while the force is decreasing. The simulation has been run until the liquid overflows, i.e. when $\beta \approx 60^{\circ}$. The behaviour of both models is quite similar however there is a small difference between the analytical model (dashed line) and the numerical model (solid line). The force values corresponding to the maximal filling angles are respectively equal to $97 \mu \mathrm{N}$ for the $\Phi 0.3 \mathrm{~mm}$ gripper and to $168 \mu \mathrm{N}$ for the $\Phi 0.5 \mathrm{~mm}$ gripper.

Finally, direct force measurements are shown in figure 7 , for 4 different grippers $(B, F, H, J)$. In each case, the gripper has been dipped to liquid and put in contact with ball to handle (the ball diameter is equal to that one of the gripper). This first contact discards the main part of liquid so that the volume of liquid becomes smaller than the conical cavity of the grippers (henceforth, it cannot be seen with the camera). Then, the gripper is applied onto the ball $n$ times without refilling it and the capillary force between the gripper and the ball is measured. After each rupture of the meniscus (i.e. after each contact), a bit of liquid is left on the ball, so that the volume of liquid involved in the

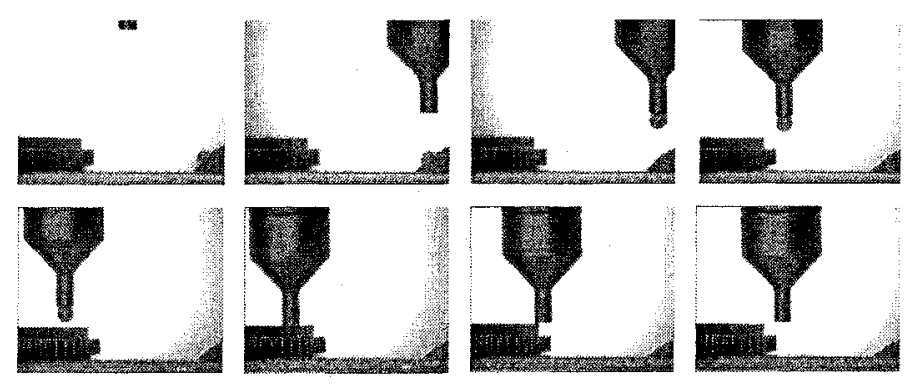

Figure 5. Assembly sequence: the diameter of the handled ball is $0.5 \mathrm{~mm}$. 


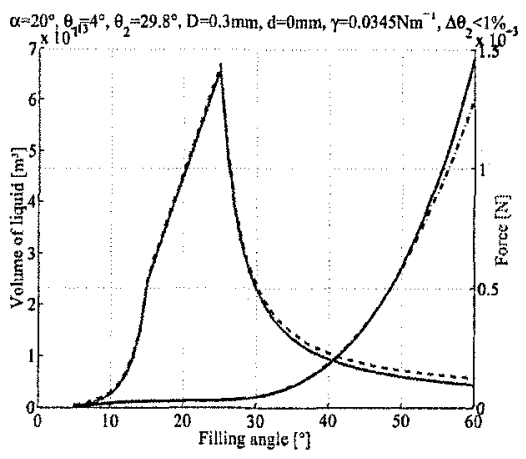

Figure $6 a$. Volume of liquid and force as a function of the filling angle $\beta$ (numerical model: solid line, analytical mode: dashed line) for the gripper $J$.

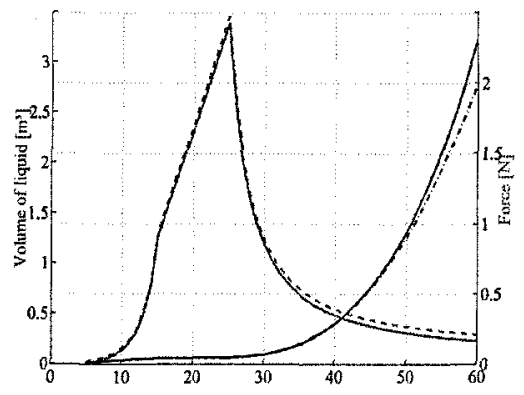

Figure $6 b . \quad$ Volume of liquid and force as a function of the filling angle $\beta$ (numerical model: solid line, analytical mode: dashed line) for the gripper $\mathrm{H}$.

manipulation is decreasing (however it cannot be measured). Therefore, according to the models, the force should increase, which can be observed in figure 7 for grippers $\mathrm{H}$ and J. For grippers $\mathrm{B}$ and $\mathrm{F}$, the simulation cannot be run because the effects of the channel have not been modelled. Nevertheless, the effect of this channel is to decrease the force (the force generated by larger grippers $\mathrm{B}$ and $\mathrm{F}$ is even smaller than the one generated by the smaller gripper J).

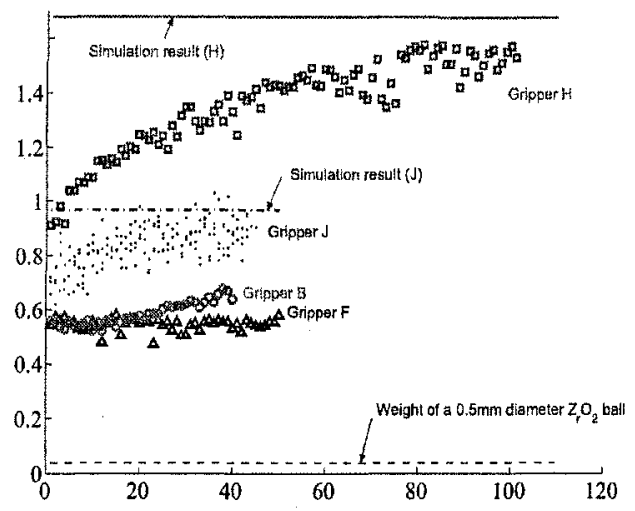

Figure 7. Force measurements. 


\section{CONCLUSION}

and Jacques Jacot

This paper presented the study of a gripper using the effects of the surface tension to handle submillimetric balls. Its originality lies in the developed design and in the adaptation of existing force models to this case study (both analytical and numerical models have been developed). A test bed has been used to experimentally validate the handling principle, showing a successfull cycle of pick and place. The comparison with experimental results validates the force models in terms of trend and order of magnitude. A further validation should require measuring the volume of the liquid inside the conical cavity of the gripper. The proposed gripper design is well adpated for $0.5 \mathrm{~mm}$ diameter balls and develops a force which is large enough too in the case of $0.3 \mathrm{~mm}$ balls. The liquid supply has been achieved by dipping the gripper tip to the handling liquid. At this step, the presence of an internal channel is not necessary to supply the liquid. Moreover, the channel decreases the force. As a concluding remark, let us note that other applications than the handling of small balls are going to be studied. On a short term, the gripper will be moved by a Sysmelec robot, in order to assess the performances of this gripping principle.

\section{ACKNOWLEDGEMENTS}

The authors wish to thank J.-J. Crausaz and P. Zbinden for the manufacturing, G. Kulik and P. Hoffmann for the coating of the prototypes.

\section{REFERENCES}

1. Bark, K.-B. (1999). Adhäsives Greifen von kleinen Teilen mittels niedrigviskoser Flüssigkeiten. Springer.

2. Biganzoli, F., Fassi, I., and Pagano, C. (2005). Development of a gripping system based on capillary force. In Proceedings of ISATP05, Montreal, Canada.

3. Grutzeck, H. and Kiesewetter, L. (1998). Downscaling of grippers for micro assembly. In Proc. of 6th Int. Conf. on Micro Electro, Opto Mechanical Systems and Components, Potsdam.

4. Lambert, P. (2004). A Contribution to Microassembly: a Study of Capillary Forces as a gripping Principle. PhD thesis, Université libre de Bruxelles, Belgium.

5. Lambert, P. and Delchambre, A. (2005). Parameters ruling capillary forces at the submillimetric scale. Langmuir, 21:9537-9543.

6. Obata, K. J., Motokado, T., Saito, S., and Takahashi, K. (2004). A scheme for micro-manipulation based on capillary force. J. Fluid Mech, 498:113-21.

7. Orr, F. M., Scriven, L. E., and Rivas, A. P. (1975). Pendular rings between solids: meniscus properties and capillary force. J. Fluid Mech., 67:723-42. 